\title{
Direct observation of patchy fluid distribution: Laboratory study
}

\author{
M. Lebedev \& B. Gurevich \\ Curtin University of Technology, Perth, Australia \\ B. Clennell, M. Pervukhina, V. Shulakova, T. Müller \\ CSIRO Petroleum, Perth, Australia
}

\begin{abstract}
The geohazards predictions during oil and gas extraction are becoming extremely important recently. Reservoir development changes fluid distribution inside reservoir and in some cases facilitates major geohazards. Exploration geophysics methods and especially seismic method are usually applied for investigation of underground fluid distribution and thus prevention of possible hazards. Interpretation of exploration seismograms requires understanding of the relationship between distribution of the fluids patches and acoustic properties of rocks. The sizes of patch as well as its distribution play a significant role in seismic response. In this paper we reported the results of laboratory experiments of simultaneous observation of fluid saturation by X-ray computer tomography and ultrasonic velocity measurements. Results are compared with numerical simulations.
\end{abstract}

\section{INSTRUCTIONS}

Maximising the recovery of known hydrocarbon reserves is one of the biggest challenges facing the petroleum industry today. Optimal production strategies require accurate monitoring of productioninduced changes of reservoir saturation and pressure over the life of the field. Time-lapse seismic technology is increasingly used to map these changes in space and time. However until now, interpretation of time-lapse seismic data has been mostly qualitative. In order to allow accurate estimation of the saturation, it is necessary to know the quantitative relationship between fluid saturation and seismic characteristics (elastic moduli, velocity dispersion and attenuation). The problem of calculating acoustic properties of rocks saturated with a mixture of two fluids has attracted considerable interest (Gist 1994; Mavko \& Holen-Hoeksema 1994; Knight et al. 1998); for a comprehensive review of theoretical and experimental studies of the patchy saturation problem see Toms et al. (2006).

For a porous rock whose matrix is elastically homogeneous, and inhomogeneities are caused only by spatial variations in fluid properties, two theoretical bounds for the P-velocity are known (Mavko \& Mukerji 1998, Mavko et al. 1998). In the static (or low-frequency) limit, saturation can be considered as homogeneous, and hence the rock may be looked at as saturated with a homogeneous mixture of the fluids. In this case the bulk modulus of the rock is de- defined by the Gassmann equation with the fluid bulk modulus given by Wood's formula i.e., the saturation-weighted harmonic average of the bulk moduli of fluids. The Gassmann-Wood bound is valid when the characteristic patch size is small compared to the fluid diffusion length. The diffusion length is primarily controlled by rock permeability, fluid viscosity and wave frequency. In the opposite case, when the patch size is much larger than the diffusion length, there is no pressure communication between fluid pockets and consequently no fluid flow occurs. In this no-flow (or high-frequency) limit, the overall rock behaves like an elastic composite consisting of homogeneous patches whose elastic moduli are given by Gassmann's theory. Since all these patches have the same shear modulus, the effective P-wave modulus can be obtained using Hill's equation, i.e., the saturation-weighted harmonic average of the P-wave moduli. Whereas the Gassmann-Wood and Gassmann-Hill bounds apply in the low- and high-frequency limits, respectively, for intermediate frequencies, uneven deformation of fluid patches by the passing wave results in local pressure gradients and hence in the wave-induced fluid flow. Such wave-induced flow cases wave attenuation and thus velocity dispersion. The effects of regularly distributed fluid patches of simple geometry (spheres, flat slabs) were first studied by White (1975), White at al. (1975), and Dutta \& Ode (1979). More recently, the effect of regularly distributed patches of more general shape was modelled by 
Johnson (2001) and Pride et al. (2004). For randomly distributed fluid patches, Müller \& Gurevich (2004) and Müller et al. (2008) showed how the effect of wave-induced flow controls the transition from the Gassmann-Wood to the Gassmann-Hill bounds.

While theoretical poroelastic models can predict the acoustic response for a given spatial distribution of fluid patches, the factors controlling the formation of the patches are less well understood. These factors can be studied using fluid-injection experiments in the laboratory. Previously reported laboratory observations demonstrate a qualitative link between fluid patch distribution and acoustic velocities (Cadoret et al. 1995, 1998; Monsen \& Johnstad 2005). In order to get a deeper insight into the factors influencing the patch distribution and the associated wave response, we perform simultaneous measurements of P-wave velocities and rock sample X-ray computer tomography (CT) imaging. The CT imaging allows us to infer the fluid distribution inside the rock sample during saturation (water imbibition). We then show that the experimental results are consistent with theoretical predictions and numerical simulations.

\section{EXPERIMENTAL SET UP}

Experiments are performed on a cylindrical sample (38 $\mathrm{mm}$ in diameter and $60 \mathrm{~mm}$ long) cut from a Casino sandstone (Otway Basin, Australia). The sample is dried at $100{ }^{\circ} \mathrm{C}$ under reduced pressure for $24 \mathrm{~h}$. The petrophysical properties are measured using a Coretest AP-608 automatic permeameter/porosimeter (Table 1). Then, the sample is sealed with a thin epoxy layer in order to prevent fluid leakage through the surface. Longitudinal (Vp) and shear wave (Vs) velocities at a frequency of $1 \mathrm{MHz}$ are measured in the direction across to the core axis (perpendicular to the fluid flow) using broadband ultrasonic transducers. Intermediate aluminum "guidepins" are placed between the sample and transducers to secure sufficient and constant coupling, as well as to provide transparency for X-ray radiation.

Two different saturation methods - referred to as dynamic and quasi-static saturation - are used in this study. In the dynamic saturation experiments, the samples are jacketed in the experimental cell made out of X-ray transparent material PMMA (Figure 1).

Distilled water is injected into the sample from one side. The injection rate is $10 \mathrm{~mL}$ per day. In quasi-static saturation experiments the samples are saturated during a long period of time (up to 2 weeks) under reduced pressure in order to achieve near-uniform fluid distribution for a given saturation level.
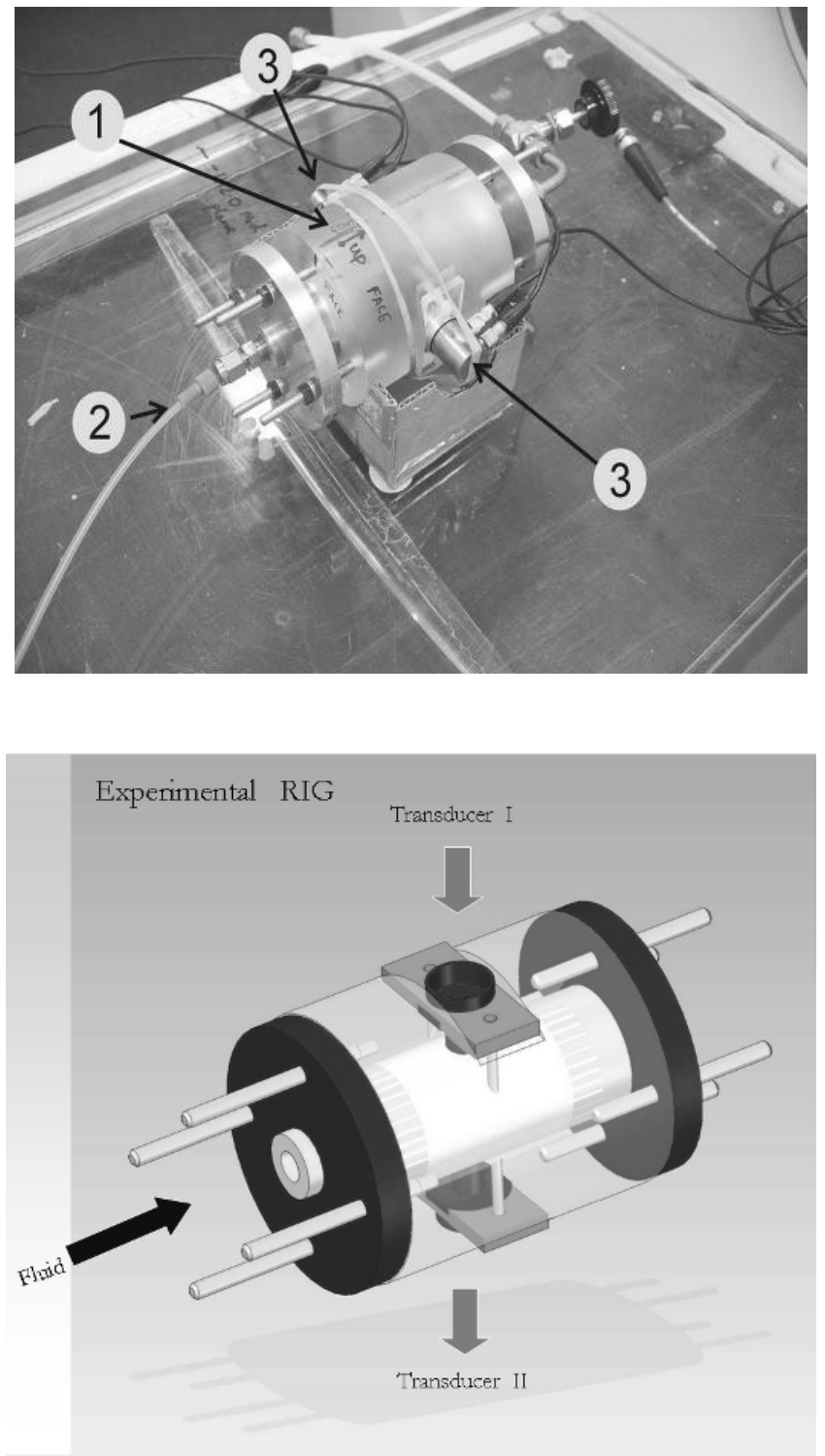

Figure 1. Core sample jacketed inside experimental rig: 1- Xray transparent jacket; 2 -injection pipe; 3 - ultrasonic transdusers.

For quasi-static experiments the saturation level is determined by measurement of the volume (weight) of water fraction divided by total volume of pores. The fluid distribution in the dynamic saturation experiments (both spatial and time dependence) is characterized using X-ray computer tomography. (Figure 2).

The resolution is $0.2 \times 0.2 \times 1 \mathrm{~mm} 3$ (voxel size). This resolution is not sufficient to image the exact fluid patch geometry; however, it shows overall character of the fluid distribution. The fluid saturation is estimated as a difference in average CTnumber (a value related to material density) between the saturated and dried sample divided by the volume of porous fraction. 


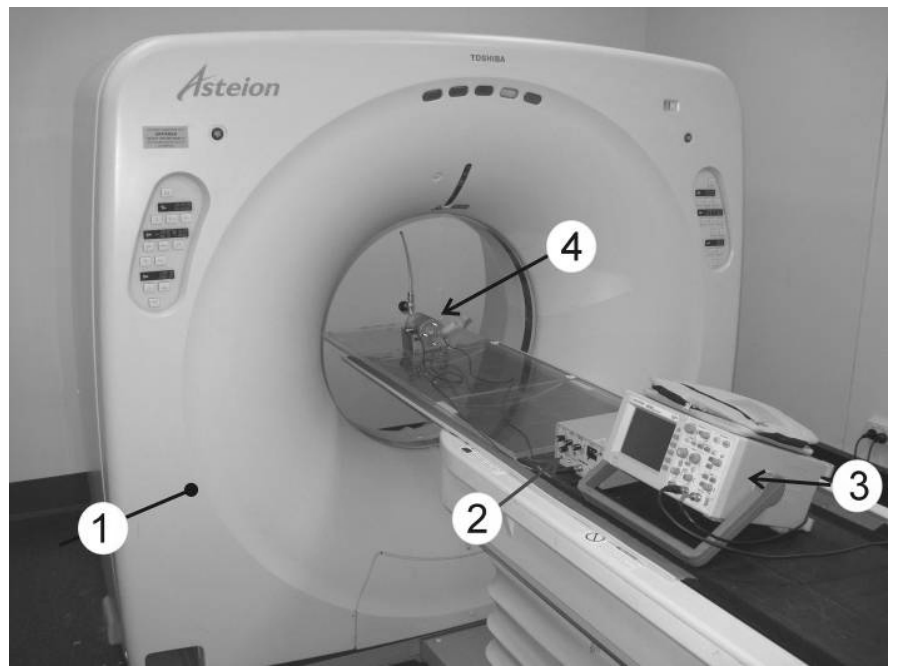

Figure 2. Simultaneous acquisitions of acoustic properties and fluid saturation using ultrasonic transducers and CT scan: 1 Computer tomograph; 2 - ultrasonic pulse-receiver; 3 - oscilloscope; 4- jacketed sample.

The temporal evolution of the saturation profile along the rock sample is shown in Figure 3. We see that the overall saturation profile becomes progressively more uniform over time. All experiments are performed at laboratory environment at a temperature of $25^{\circ} \mathrm{C}$. Velocity is obtained by first break picking on output signal. Figure 4 shows the P-wave velocity as a function of saturation for the Casino Otway sandstone from the dynamic saturation experiment (empty symbols) and quasi-static saturation experiment (solid symbols). A transition from the Gassmann-Wood to Gassmann Hill bound is clearly

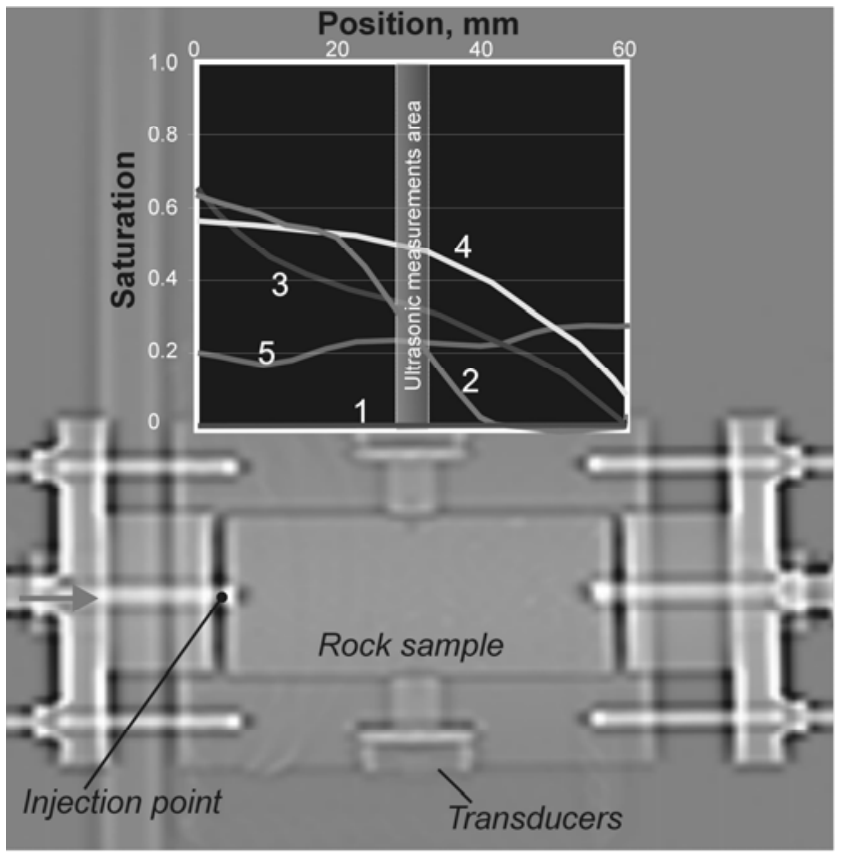

Figure 3. X-ray image of the whole core sample. The embedded plot shows the profiles of water saturation along the 60 $\mathrm{mm}$ long sample at different times (increasing from 1 to 5 ) during the saturation experiment. observed for water saturations of $40-50 \%$ for dynamic saturation and $60-70 \%$ for quasi-static saturation. Figure 5 shows CT images recorded during the dynamic saturation experiments. Scans 1-5 correspond to points 1-5 on the velocity-saturation curve (Figure 4): increasing saturation from the dried sample (1) up to $50 \%$ (point 4 ) and then decreasing saturation down to $26 \%$ (point 5). At low saturations, the velocity can be well described by the Gassmann Wood relation; however, a sharp increase in velocity is observed when saturation exceeds $40 \%$ (points 3 and 4 in the Figure 4). Then, the velocity-saturation approaches the Gassmann-Hill bound.

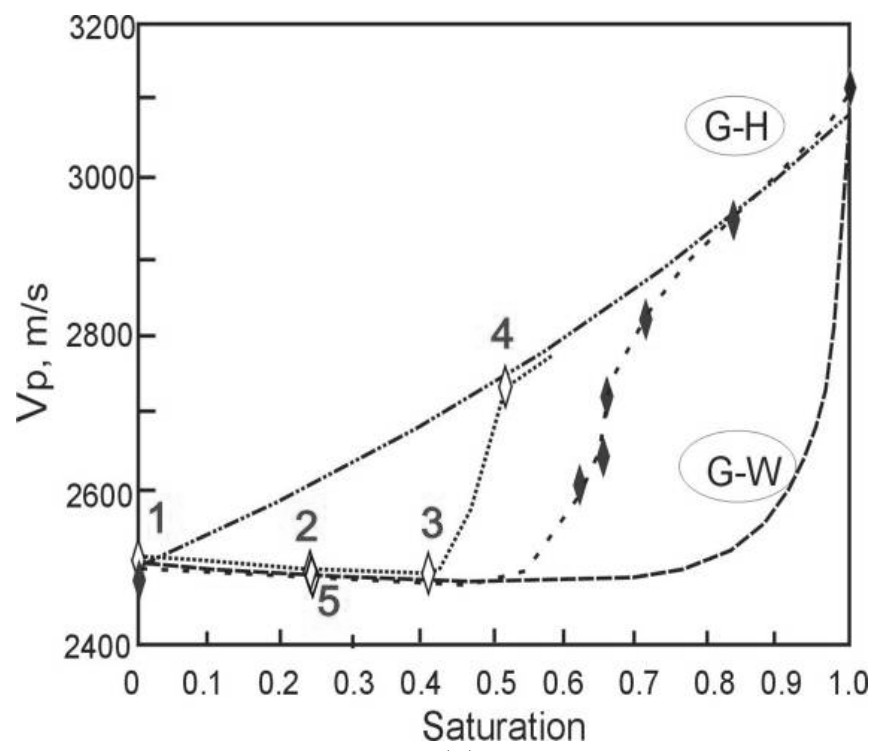

(a)

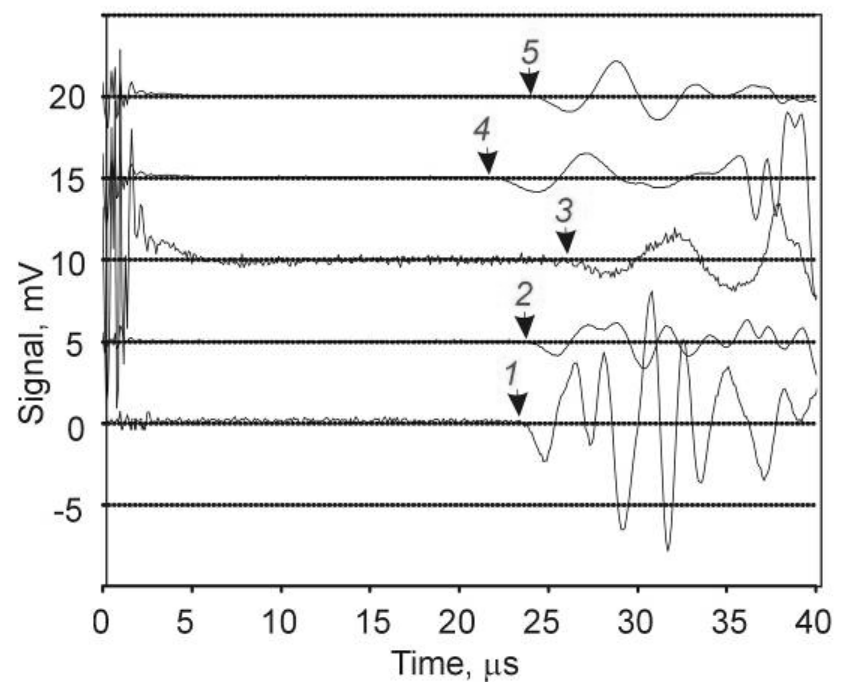

(b)

Figure 4. a) Velocity versus saturation for the Casino Otway Basin sandstone from the quasi-static saturation experiment (black diamonds) and dynamic saturation experiment (white diamonds). The numbers from 1 to 5 indicate acoustic measurements corresponding to the CT images shown in Figure 5, and saturation profiles shown in Figure 3. Theoretical Gassmann-Wood (G-W) and Gassmann-Hill (G-H) bounds are shown by dashed and dash-dotted lines, respectively. b) Output signals corresponding to five stages during the dynamic fluid injection experiment. 


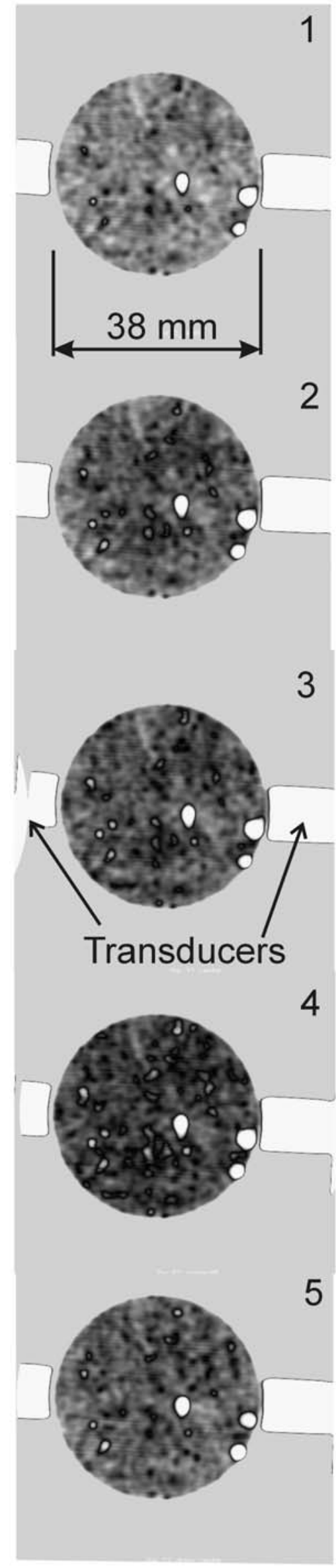

We also observe that for relatively large fluid injection rates (dynamic experiment) the transition from homogeneous to patchy saturation (from the Gassmann-Wood to Gassmann-Hill) occurs at smaller degrees of saturation as compared with low injection rates (quasi-static experiment). This observation suggests that the process of fluid patch formation is controlled by the injection rate.

\section{NUMERICAL SIMULATIONS}

In order to validate our interpretation of the velocitysaturation relation in terms of wave-induced flow we also perform numerical simulations of wave propagation in a 2-D poroelastic solid. The simulations are performed using 2D finite-difference solver of Biot's equations of dynamic poroelasticity (Krzikalla \& Müller 2007).

We numerically simulate plane wave propagation through 2D water saturated media with circle gas inclusion (Figure 6b). Wave propagation is initiated by applying a normal stress at the top of the domain. As a loading function we use $\sin (3 x)$, where the duration of source signal (1 s) and time of wave propagation (5 s with $2 \cdot 10^{-4} \mathrm{~s}$ time step) are chosen sufficiently long to model quasistatic conditions and avoid resonance. The shape of outer water-saturated medium is a circle for analytical solution and a square for computational model. The size of model is 40 x 40 grid points. Periodical boundary conditions (PBC) are applied in lateral direction. Receiver configuration consists of 20 receiver lines with 20 receivers in each line. Distance between lines and receivers - 2 grid points. Material properties for the numerical model are shown in the Table 1.

Table 1. Model parameters fo 2D medium.

$\begin{array}{lcll} & \text { Rock frame } & \text { Gas } & \text { Fluid } \\ \text { Porosity } & 0.2 & & \\ \begin{array}{l}\text { Permeability }\left(\mathrm{m}^{2)}\right. \\ \text { Bulk modulus }\end{array} & 1 \cdot 10^{-12} & & \\ \mathrm{~K}_{\text {dry }}(\mathrm{Pa}) & 5 \cdot 10^{9} & 0.1 \cdot 10^{9} & 2.25 \cdot 10^{9} \\ \mathrm{~K}_{\text {grain }}(\mathrm{Pa}) & 35 \cdot 10^{9} & & \\ \text { Shear modulus } & & & \\ \mathrm{G}_{\text {dry }}(\mathrm{Pa}) & 11 \cdot 10^{9} & & \\ \text { Density }\left(\mathrm{kg} / \mathrm{m}^{3}\right) & 2650 & 100 & 990 \\ \text { Viscosity }(\mathrm{Pa} \cdot \mathrm{s}) & & 0.1 \cdot 10^{-6} & 1 \cdot 10^{-3}\end{array}$

Figure 5. CT images of the Casino Otway Basin sandstone at different times after start of injection at the same position: 1 dried sample; $2-1 \mathrm{~h} ; 3-24 \mathrm{~h} ; 4-72 \mathrm{~h}$. Contour of guide-pins are visible at right- and left-hand side of the sample. 

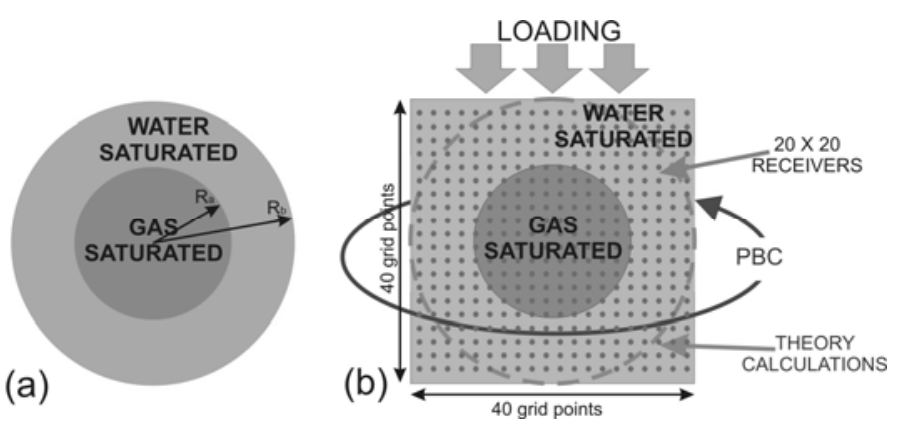

Figure 6. Schematic plot of patchy-saturated model: a 2D periodic geometry with inner circle filled with gas and outer medium ( $\mathrm{a}$ - circle for analytical solution and b - square for computer model) filled with water.

As a result of numerical simulations we obtain seismograms of velocity divergence $v_{x x}+v_{y y}$, velocity difference $\mathrm{v}_{\mathrm{yy}}-\mathrm{v}_{\mathrm{xx}}$, and stresses $-\mathrm{S}_{\mathrm{xx}}-\mathrm{S}_{\mathrm{yy}}, \mathrm{S}_{\mathrm{yy}}-\mathrm{S}_{\mathrm{xx}}$. In order to obtain frequency-dependent velocity and attenuation curves we used the method suggested by Masson (2007). At first, integration of the bulk velocity divergence and velocity difference yields mean and differential strains. Then, stresses and strains are averaged within the model and transformed to the frequency domain by Fourier transform. Finally, effective bulk and shear moduli, fast P-wave velocity and attenuation are calculated from these averaged fields according to Wenzlau (in press)

Velocity dispersion and attenuation are numerically simulated for a wide range of frequencies from $10^{-2}-10^{3} \mathrm{~Hz}$. The numerical results for water saturation of 0.75 are shown by black dots in Figure 7 in comparison with the analytical solutions for three slightly different media..

The analytical results for 3 different media are shown because the analytical and numerical results are obtained for similar but still slightly different models (Figure 6a,b) where the model at Figure 6a does not fill a plane.

Dispersion and attenuation curves (dots) for four frequencies $(0.4,0.6,1,2 \mathrm{~Hz})$ versus water saturation are shown in the Figure 8 together with analytical solution calculated with the same $\mathrm{S} / \mathrm{V}$ ratio (the ratio of the boundary area between two phases to the sample volume). Variations in water saturation $(0.19,0.44,0.64,0.75,0.84,0.94)$ are obtained by variation of the inner circle radius. Velocities are bounded by Biot-Gassmann-Wood (BGW) curve for low frequencies and by Biot-Gassmann-Hill (BGH) curve for high frequencies Johnson (2001).
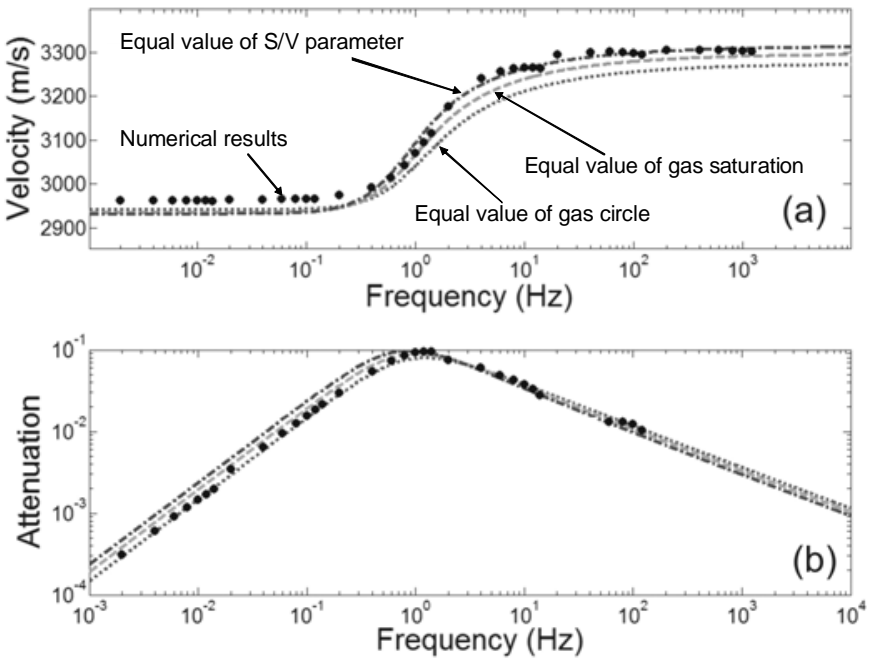

Figure 7. Three variants of theoretical prediction (dashed lines) and numerical calculations (dots) of P- wave velocity (a) and attenuation (b) vs. frequency.
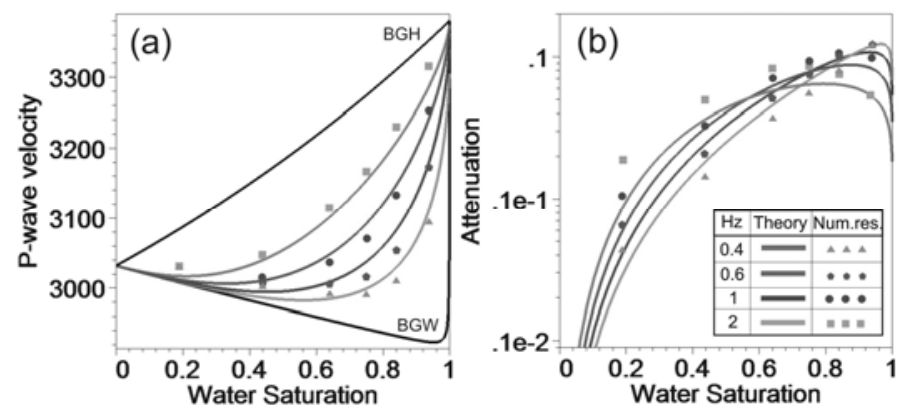

Figure 8. Theoretical prediction (solid lines) and numerical calculations (dots) of P- wave velocity (a) and attenuation (b) vs. water saturation for different frequencies.

Figures 7 and 8 show that our numerical results are in a reasonably good agreement with the theory. The slight existing discrepancies between the numerical and analytical results are most probably caused by different geometries rather than having a physical significance.

Figure 9 shows the modeled velocity-saturation relation obtained for material constants and wave frequency used in laboratory experiment described above. The degree of saturation is varied by increasing the size of the randomly distributed fluid patches. It can be seen that for large saturations, the fluid patches begin to cluster resembling the clustering observed in the CT scans. Despite the simplified numerical setup (2D modeling only and patch distribution not derived directly from the CT scans) the simulation results reproduce the overall behavior of the measured velocity-saturation relation. 


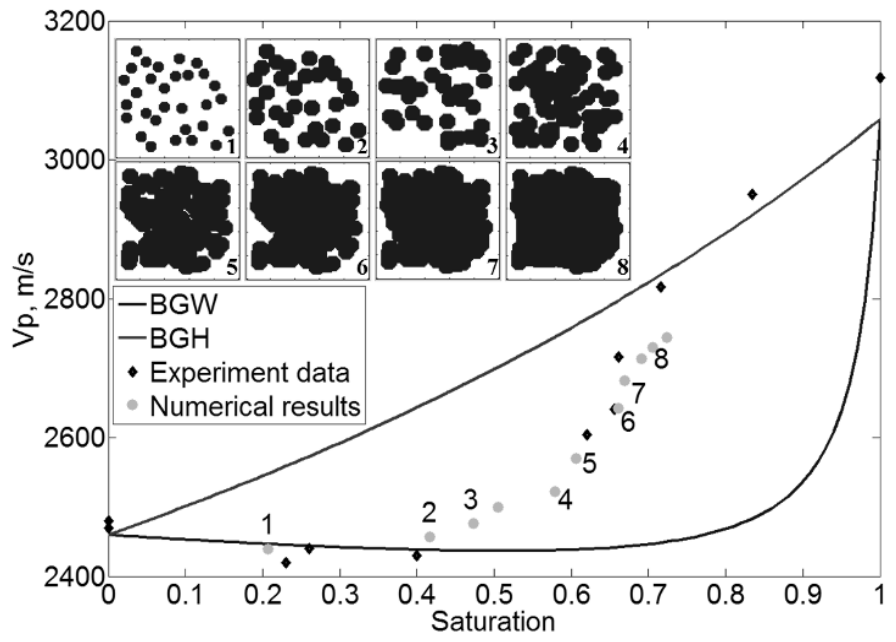

Figure 9. Velocity-saturation relation determined from numerical simulations of wave propagation in a poroelastic solid with randomly distributed patches that cluster for larger saturation values (see inset). Experimentally determined velocities for the quasi-static injection experiment are also shown.

This confirms that the transition observed in the ultrasonic measurements can be attributed to the mechanism of wave-induced flow. We plan to extend our numerical simulations to $3 \mathrm{D}$ so that the $3 \mathrm{D}$ patch distributions reconstructed from CT images can be directly used as input model in our simulations.

\section{CONCLUSIONS}

The experimental results obtained on lowpermeability samples show that at low saturation values the velocity-saturation dependence can be described by the Gassmann-Wood relationship. At intermediate saturations there is a transition behavior that is controlled by the fluid patch arrangement and fluid patch size. Also, the fluid patch size is controlled by the injection rate. In particular, we show that for relatively large fluid injection rate this transition occurs at smaller degrees of saturation as compared with high injection rate.

The results illustrate the non-unique relationships between saturation and velocity in sandstones dependent on texture and fluid displacement history: fuller understanding of these phenomena are needed for accurate assessment of time lapse seismic measurements, be they for oil and gas recovery or for $\mathrm{CO}_{2}$ disposal purposes.

\section{REFERENCES}

Cadoret, T., Marion D. \&. Zinszner B. 1995. Influence of frequency and fluid distribution on elastic wave velocities in partially saturated limestones. Journal of Geophysical Research 100: 9789-9803.

Cadoret, T., Mavko, G. \& Zinszner, B. 1998. Fluid distribution effect on sonic attenuation in partially saturated limestones. Geophysics 63: 154-160.

Dutta, N. C. \& Odé, H. 1979. Attenuation and dispersion of compressional waves in fluid-filled porous rocks with partial gas saturation (White model)—Part I: Biot theory. Geophysics 44: 1777-1788.

Gassman, F. 1951. Elastic waves through a packing of spheres. Geophysics 16: 673-685.

Gist, A.G. 1994. Interpreting laboratory velocity measurements in partially gas-saturated rocks. Geophysics 54: 1100-1108.

Johnson, D. L. 2001. Theory of frequency dependent acoustics in patchy-saturated porous media. Journal of the Acoustical Society America 110: 682- 694.

Knight, R., Dvorkin, J. \& Nur, A. 1998. Acoustic signatures of partial saturation. Geophysics 63: 132-138.

Krzikalla, F. \& Müller, T.M. 2007. High-contrast finitedifferences modeling in heterogeneous poroelastic media. 77th SEG Annual Meeting, San Antonio, Extended Abstracts: $2030-2034$.

Masson, Y.J. \& Pride, S.R. 2007. Poroelastic finite-difference modeling of seismic attenuation and dispersion due to mesoscopic-scale heterogeneity. Journal of Geophysical Research -Solid Earth 112: B03204.

Mavko, G. \& Nolen-Hoeksema, R. 1994. Estimating seismic velocities at ultrasonic frequencies in partially saturated rocks. Geophysics 59: 252-258.

Mavko, G. \& Mukerji, bT. 1998. Bounds on low frequency seismic velocities in partially saturated rocks. Geophysics 63: 918-924.

Mavko, G., Mukerji, T. \& Dvorkin, J. 1998. The Rock Physics Handbook: Tools for seismic analysis in porous media. Cambridge: Cambridge University Press.

Monsen, K. \& Johnstad, S. E. 2005. Improved understanding of velocity-saturation relationships using 4D computertomography acoustic measurements. Geophysical Prospecting 53: 173-181.

Müller, T. M. \& Gurevich, B. 2004. One-dimensional random patchy saturation model for velocity and attenuation in porous rocks. Geophysics 69: 1166-1172.

Müller, T. M., Toms-Stewart, J. \& Wenzlau, F. 2008. Velocitysaturation relation for partially saturated rocks with fractal pore fluid distribution. Geophysical Research Letters 35: L09306.

Pride, S.R., Berryman, J.G. \& Harris, J.M. 2004. Seismic attenuation due to wave- induced flow. Journal of Geophysical Research 109: B01201.

Toms, J., Müller, T.M., Ciz, R. \& Gurevich, B. 2006. Comparative review of theoretical models for elastic wave attenuation and dispersion in partially saturated rocks. Soil Dynamics and Earthquake Engineering. 26: 548-565.

Wenzlau, F., \& Müller, T.M. (in press). Finite-difference modeling of wave propagation and diffusion in poroelastic media. Geophysics.

White, J. E. 1975. Computed seismic speeds and attenuation in rocks with partial gas saturation. Geophysics 40: 224-232.

White, J. E., Mikhaylova, N. G. \& Lyakhovitskiy, F. M. 1976. Low-frequency seismic waves in fluid-saturated layered rocks. Physics of the Solid Earth Transactions. Izvestiya 11: 654-659. 